\title{
Enhanced susceptibility of CA3 hippocampus to prenatal nicotine exposure
}

\author{
O. O. Kalejaiye ${ }^{1,2}$ and M. C. Gondré-Lewis ${ }^{1,2 *}$ \\ ${ }^{1}$ Laboratory for Neurodevelopment, Department of Anatomy, Howard University College of Medicine, Washington, DC, USA \\ ${ }^{2}$ Neuropsychopharmacology Laboratory, Department of Psychiatry and Behavioral Sciences, Howard University College of Medicine, Washington, DC, USA
}

\begin{abstract}
The brain is highly susceptible to adverse effects of drugs of abuse during early phases of life. Prenatal nicotine exposure (PNE), a preventable cause of gestational and infant mortality, can alter neuron wiring and induce sustained deficits in attention and learning. Here, a rat model of PNE (embryonic days 7-21) was used to examine the maturing hippocampus, which encodes new memories and processes emotional memory. Components of synaptic signaling were evaluated at postnatal day 14 (P14), a period of prolific synaptogenesis in rats, to determine if glutamatergic transmission-associated molecules are regulated in subregions of hippocampus as early as P14. PNE resulted in reduced expression of GluN2B, GluA2 and CaMKII $\alpha$, but elevated SNAP25 proteins specifically in the CA3 but not CA1. Only CaMKII $\alpha$ was regulated in dentate gyrus at this age. These results suggest that glutamatergic and synaptic dysregulation of learning and memory may occur in hippocampus in a temporally and subregionally specific manner.
\end{abstract}

Received 1 October 2016; Revised 17 November 2016; Accepted 27 November 2016; First published online 28 December 2016

Key words: animal, developmental stage, fetus/foetus, maternal pregnancy, outcome/system, small animals

\section{Introduction}

Smoking during pregnancy remains a leading preventable cause of gestational complications and infant mortality. The Centers for Disease Control reports that $20 \%$ of women admittedly use tobacco during pregnancy, ${ }^{1}$ and this number may indeed be higher in different parts of the world. Thus, nicotine's deleterious effects are a major public health concern. During the early neurodevelopmental period, the brain is highly susceptible to adverse effects of drugs of abuse, which shape behavioral and neurochemical profiles of the offspring. Many studies now support the positive correlation between prenatal nicotine exposure (PNE) and cognitive defects involving disrupted hippocampal circuitry ${ }^{2-7}$ and manifested in behavioral deficiencies. $^{4,6,8}$

The hippocampus, necessary for learning, memory, and long-term potentiation (LTP) of synaptic inputs, transduces its functions through distinctive subregions and cell types. The dentate gyrus (DG) is predominantly involved in the acquisition and processing of spatial and episodic memories ${ }^{9}$ and exploration of novel environments. ${ }^{10}$ The DG's functions and flexibility are likely correlated to its ability to undergo neurogenesis into adulthood. ${ }^{11,12}$ Of the Cornu Ammonis (CA) regions, $\mathrm{CA} 3$ is involved in representing episodic

*Address for correspondence: Dr M. C. Gondré-Lewis, Associate Professor, Laboratory for Neurodevelopment, Department of Anatomy, Director, Neuropsychopharmacology Laboratory, Department of Psychiatry and Behavioral Sciences, Howard University College of Medicine, 520 W Street, NW, Washington, DC 20059, USA.

(Email Mgondre-lewis@howard.edu) memories, ${ }^{13-15}$ whereas CA1 functions mainly in memory for the order of spatial ${ }^{16}$ and non-spatial stimuli. ${ }^{17-19}$ These subregions exhibit extensive connectivity in the hippocampus and with cortical and subcortical regions. For instance, CA3 receives significant direct mossy fiber inputs from the $\mathrm{DG}^{20}$ and entorhinal cortex ${ }^{21}$ and projects Schaeffer collaterals to the CA1. ${ }^{22}$ Therefore, the dysregulation of the neurochemical stoichiometry of one hippocampal subregion may have a lasting impact on the others either directly or indirectly.

Glutamatergic receptors, including $N$-methyl-Daspartate (NMDAR) and $\alpha$-amino-3-hydroxy-5-methyl-4isoxazolepropionic acid receptor (AMPAR), constitute the predominant excitatory receptor class in the hippocampus. They are responsible for strengthening of synapses and are critical for spatial learning and the formation of new memories. $^{23-26}$ In fact, a reduction of AMPA receptors resulted in reduced acquisition of new memories and the inability to recount old memories. ${ }^{27,28}$ Although the roles of NMDAR and AMPAR in learning and memory have been extensively investigated in general albeit less extensively in relation to gestational nicotine, the disproportionate effects on hippocampal subregions associated with gestational exposure of the brain to nicotine are poorly understood. Our previous findings suggest that PNE can regulate the glutamatergic signaling system during postnatal development by enhancing or inhibiting availability of AMPAR and NMDAR in the hippocampus. ${ }^{29}$ In the present study, we investigated whether prenatal nicotine regulates AMPAR and NMDAR stoichiometry and functionality, specifically in the DG, CA1 or CA3 subregions of hippocampus as early as P14 (infancy equivalent age). 


\section{Materials and methods}

\section{Animals and prenatal nicotine treatment}

This study was approved by Howard University's Animal Care and Use Committee. Timed-pregnant Sprague-Dawley rats (Harlan Laboratories, Frederick, MD, USA) weighing 250-300 g were implanted on gestational day 7 (G7) between the scapulae with an infusion pump (model \#2004) (Alzet Inc., Cupertino, CA, USA) containing either $0.9 \%$ saline (Control) or (-)-nicotine hydrogen tartrate (Sigma-Aldrich, St. Louis, MO, USA), delivered at a rate of $4 \mathrm{mg} / \mathrm{kg} / \mathrm{d} .{ }^{3}$ This dose is commonly used to mimic circulating blood nicotine levels of a 2-4 packs/ day smoker. All pumps hold a volume of $-250 \mu$ and have a flow rate of $\sim 0.5 \mu \mathrm{l} / \mathrm{h}$. On G21, pumps were removed using aseptic surgery. Surgery did not adversely affect pup birth rate or litter size. Previously published data from our laboratory indicate that pups exposed prenatally to nicotine weighed more than controls, a difference that was maintained through adulthood. ${ }^{30}$

\section{Tissue collection}

At P14, animals were anesthetized with isoflurane. Whole brains were removed from the cranial cavity, flash frozen on dry ice and stored at $-80^{\circ} \mathrm{C}$ until ready for use. Brains were sectioned at $300 \mu \mathrm{m}$, sections were mounted on glass slides, and with the aid of a dissecting microscope, $1 \mathrm{~mm}$ micro-punches were collected from CA1, CA3 and DG subregions (all three layers of each) along the anterior-posterior axis, into frozen Eppendorf tubes.

\section{Reagents and antibodies}

Nicotine was prepared fresh on the day of pump implantation in $0.9 \%$ saline, and the $\mathrm{pH}$ was adjusted to 7.4-7.6. Antibodies against the following proteins were used: calcium/calmodulin kinase II $\alpha$ (CaMKII $\alpha)(0.1 \mu \mathrm{g} / \mathrm{ml}$, ab2725), GAPDH $(0.25 \mu \mathrm{g} / \mathrm{ml}$, ab9485) (Abcam Inc., Cambridge, MA, USA), Calmodulin (CaM) $(0.07 \mu \mathrm{g} / \mathrm{ml}, 05-173)$, GluA1 $(0.17 \mu \mathrm{g} / \mathrm{ml}, \mathrm{AB} 1504)$, GluA2 (0.17 $\mu \mathrm{g} / \mathrm{ml}, \mathrm{AB} 1768)$, NR1 (1:10 000, AB9864), GluN2A and GluN2B $(0.05 \mu \mathrm{g} / \mathrm{ml}, \mathrm{AB} 1555 \mathrm{P}$ and AB1557P), SNAP25 $(0.25 \mu \mathrm{g} / \mathrm{ml}$, SL3730) (Enzo Life Sciences - Biomol) and PSD95 (1/3000, \#2507) (Cell Signaling Technology).

\section{Western blotting}

Homogenates of the dissected micro-punched tissues were made in ice cold lysis buffer [20 mM Tris-buffer, $1 \mathrm{mM}$ EDTA, $150 \mathrm{mM} \mathrm{NaCl}, 1 \%$ Triton X-100 (v/v), 0.1\% SDS, $1 \mathrm{mM}$ DTT and 1:50 protease inhibitor cocktail (Roche Diagnostics Corp, Indianapolis, IN, USA)]. Equal amounts of protein were separated using 4-12\% SDS-PAGE NuPAGE Bis-Tris gels (Invitrogen, Inc.) and transferred onto a nitrocellulose membrane. The membranes were blocked with 5\% nonfat milk in tris-buffered saline (TBS) for $1 \mathrm{hr}$ and incubated at $4^{\circ} \mathrm{C}$ overnight with primary antibody. This was followed by HRP-conjugated secondary antibody (1:3000 in TBS, Bio-Rad Laboratories, CA, USA) and exposure to chemiluminescent reagents (Bio-Rad Laboratories). The intensity of protein bands was quantified using the ImageQuant LAS 4000 series (GE Healthcare Life Sciences).

\section{Statistical analysis}

A one-way ANOVA was utilized to analyze statistics of protein expression. Where the $F$-test showed a difference, a NewmanKeuls multiple comparison post-hoc test was applied for further comparisons. The descriptive statistics are displayed as mean \pm standard error of the mean (S.E.M.).

\section{Results}

To investigate hippocampal region-dependent mechanisms of attention and learning dysfunction induced by chronic PNE, we assayed the CA3 hippocampal subregion for expression of AMPA receptor subunits (GluA1 and GluA2), necessary for the proper assembly and membrane insertion of AMPARs. We also evaluated NMDA receptor subunits (GluN2A, GluN2B, GluN2C and GluN1), important components for proper NMDAR assembly and function. Our findings show that PNE significantly and specifically mitigated expression of GluN2B (Fig. 1a) and GluA2 (Fig. 1b) in the CA3 $(P<0.05)$ but had no significant effect on GluN1, GluN2A, GluN2C (Fig. 1a) or GluA1 (Fig. 1b) when compared with control, saline-treated rats, $P>0.05$.

Next, we investigated if non-receptor components of the synaptic machinery are modulated. CaMKII $\alpha$, a major functional molecule dubbed the 'memory' molecule, mediates receptor phosphorylation and insertion at the postsynapse. ${ }^{31}$ PNE resulted in a reduction of CaMKII $\alpha$ in the CA3 (Fig. 1c) $(P<0.05)$. Interestingly, nicotine treatment caused an increase in CA3 SNAP25 $(P<0.05)$ expression (Fig. 1c), possibly indicating enhanced presynaptic terminal activity. By contrast, CaM and PSD95, other components of the postsynaptic machinery, were not detected as regulated by PNE in CA3, $P>0.05$.

The dentate gyrus (DG), is a late developing structure of the hippocampus which continues to generate new neurons into adulthood. In the DG at P14, PNE not significantly alter mean expression of NMDA receptor subunits (Fig. 2a) GluN1, GluN2A, GluN2B, GluN2C, nor of AMPAR subunits (Fig. 2b) GluA1 and GluA2, nor the pre/post synaptic proteins (Fig. 2c) SNAP25, PSD95 and CaM. CaMKII $\alpha$ was the only postsynaptic protein detected as modulated in the DG at P14 (Fig. 2c), with a net reduction in expression in the DG of PNE prenatal nicotine exposed rats $(P<0.05)$.

Analysis of CA1 revealed no detectable effect of PNE on the expression of NMDA receptors (Fig. 2d); AMPA receptors (Fig. 2e), or pre/post synaptic proteins (Fig. 2f); $(P>0.05)$.

\section{Discussion}

Here, we report that gestational exposure to nicotine through the pregnant dam, modulates glutamatergic neurotransmission by altering all aspects of synaptic communication, resulting in 

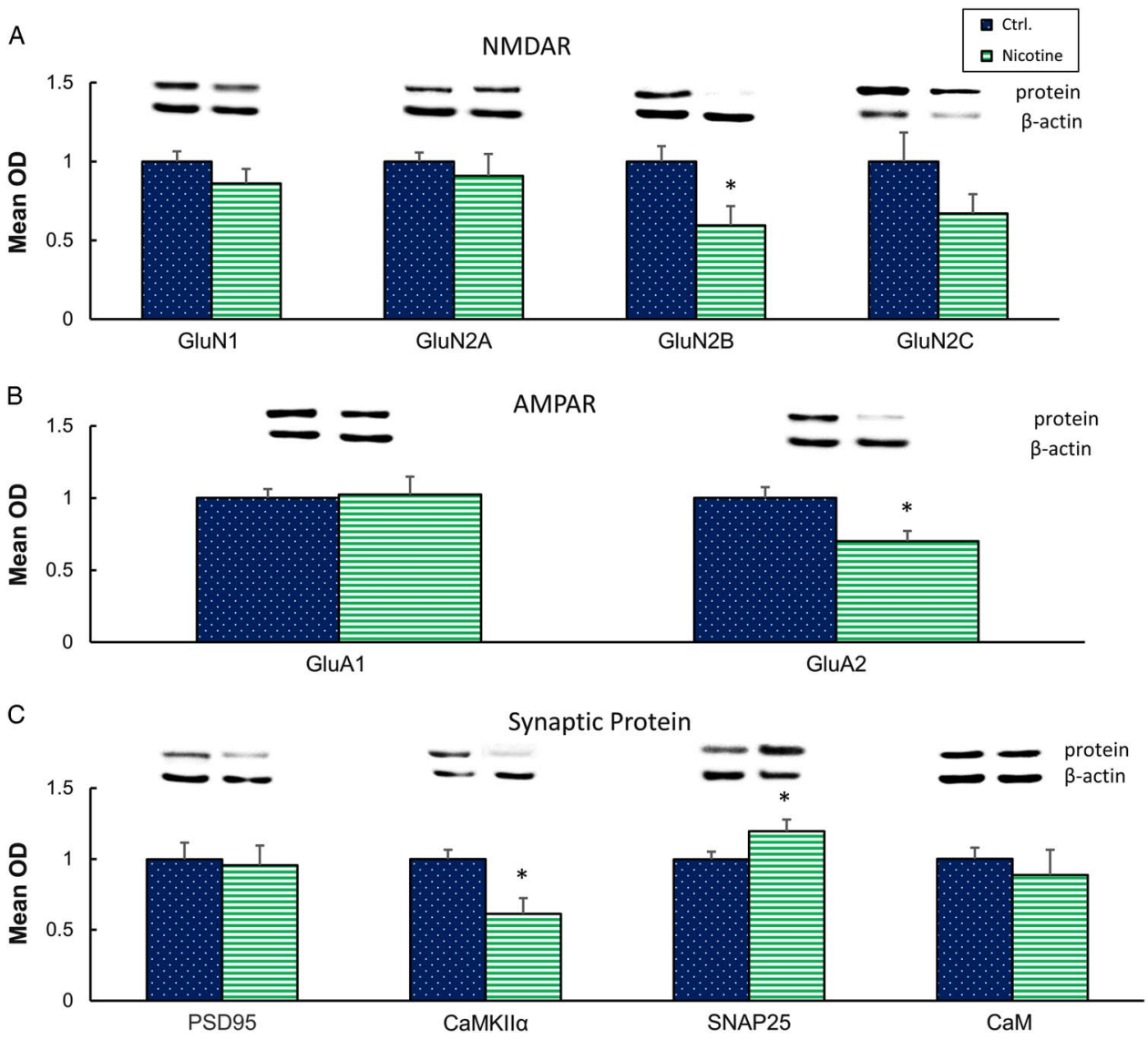

Fig. 1. Effects of prenatal nicotine exposure on synaptic proteins of CA3. (a) NMDAR subunits GluN1, GluN2A, GluN2B, GluN2C, (b) AMPAR subunits GluA1 and GluA2 and (c) soluble postsynaptic proteins, PSD95, CaMKIIIa, CaM and presynaptic SNAP25, quantified after Western blotting. *, $P<0.05 ; n=4-10$. NMDAR, $N$-methyl-D-aspartate; AMPAR, $\alpha$-amino-3-hydroxy-5-methyl4-isoxazolepropionic acid receptor.

increased expression of SNAP25 localized to presynaptic vesicles, reduction of NMDA and AMPA receptor recruitment, and reduction of postsynaptic machinery components, specifically in the CA3. We further report early dysregulation at P14 of the developing DG's postsynaptic network via reduction of CaMKII $\alpha$. This perturbation of synaptic proteins as investigated, was absent in the CA1 altogether at this age. Our findings implicate a CA3-mediated dysregulation of glutamatergic neurotransmission as a neurochemical path through which premature activation of hippocampal cholinergic afferents exert their effect on learning, memory and behavior.

The P14 neurodevelopmental period was strategically chosen because it corresponds to the phase in which hippocampal CA neurons differentiate, stabilize and strengthen their synaptic contacts, and when the tertiary dentate matrix, responsible for the increase in granule cell population, is operating at peak performance; that is, still actively proliferating. ${ }^{32,33}$ PNE is known to disrupt neuroanatomical connections of the developing brain and to have long lasting effects on the neurochemistry and neurophysiology of adult brains. ${ }^{2,3,33}$

In the current study of P14 brains, GluA2 receptors were downregulated in the CA3 whereas previous analysis of whole homogenate of P14 hippocampus from PNE rats showed both GluA1 and GluA2 as statistically indistinguishable from controls. ${ }^{29}$ These current data suggest that modulation of AMPAR stoichiometry and expression in a CA3-specific manner may partially explain PNE-mediated reductions in learning new tasks and memory retrieval. ${ }^{34}$ The CA3 subregion, important for associative learning, has been shown to be sensitive to prenatal perturbations, consistent with developmental atrophy of its pyramidal dendrites following prenatal stress. ${ }^{35}$ Neuronal projections which originate in the CA3 project onto CA1 as well as the lateral septal nuclei and the vertical limb of the diagonal band of Broca which reciprocally modulate hippocampal function. ${ }^{36-38}$ Interestingly, CA3 pyramidal cell collaterals project back onto the DG as well. ${ }^{36,39}$ Thus, the effects of premature activation of nicotinic receptors may not only disrupt rapid encoding of spatial information in short-term and episodic memory, but may also interfere with the functions of CA3 efferent targets such as cerebral cortex. Such functions include but are not limited to reward, reinforcement, adaptability to stress and neurogenesis as reported in models of disruptive environmental exposures during early life. ${ }^{3,40,41}\left[{ }^{3} \mathrm{H}\right]$ AMPAR binding studies in adult rat hippocampus exposed to 

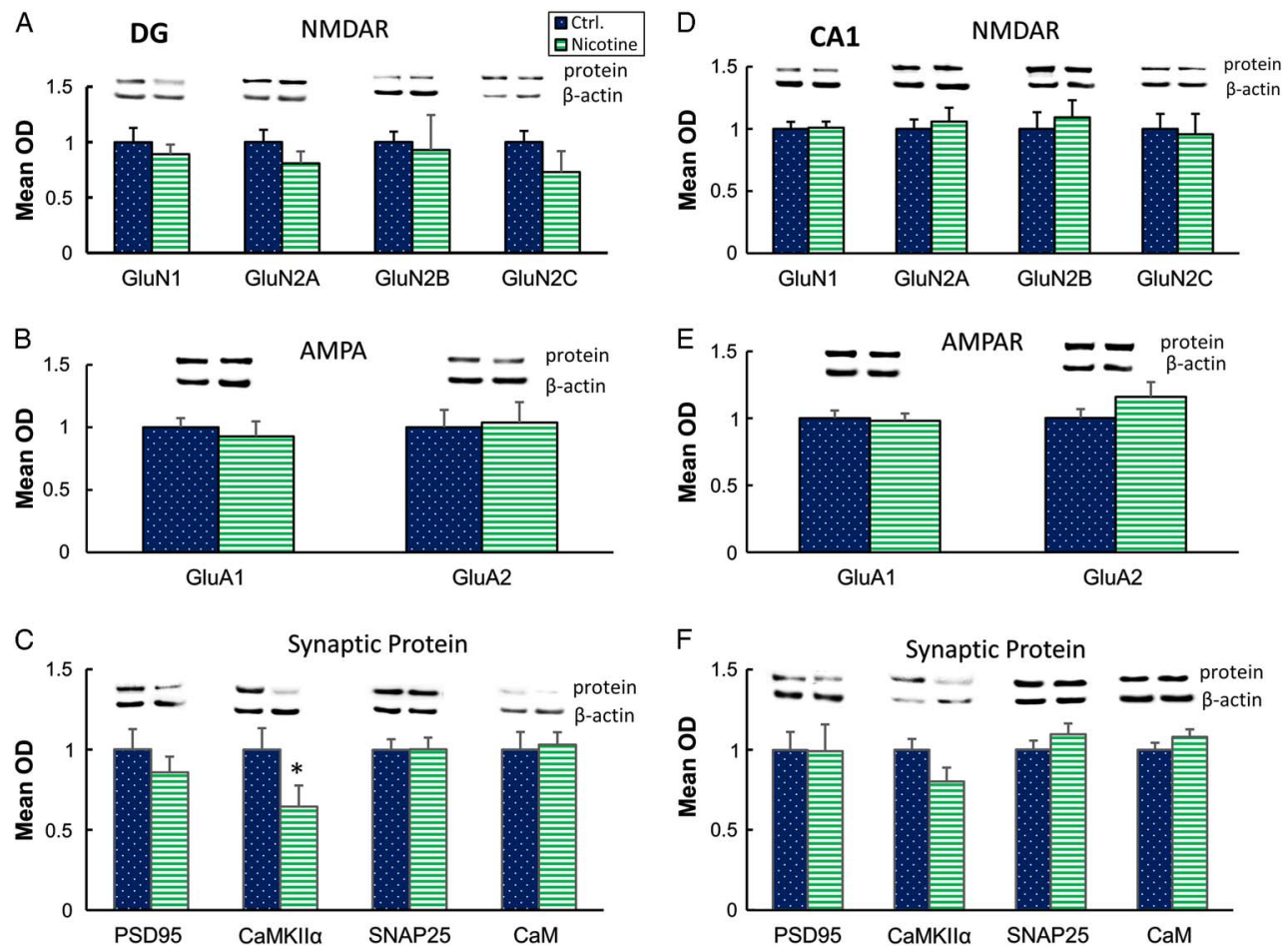

Fig. 2. Effects of prenatal nicotine exposure on hippocampal DG and CA1 synaptic proteins. Left, DG expression of (a) NMDAR subunits GluN1, GluN2A, GluN2B, GluN2C, (b) AMPAR subunits GluA1 and GluA2 and (c) postsynaptic proteins, PSD95, CaMKIIa, CaM and presynaptic SNAP25. Right, CA1 expression of (d) GluN1, GluN2A, GluN2B, GluN2C, $(e)$ GluA1 and GluA2 and ( $f$ ) PSD95, CaMKIIa, $\mathrm{CaM}$ and SNAP25. ${ }^{*} P<0.05 ; n=4-10$ per group. DG, dentate gyrus; NMDAR, $N$-methyl-D-aspartate; AMPAR, $\alpha$-amino-3-hydroxy-5methyl-4-isoxazolepropionic acid receptor.

$\mathrm{PNE}^{29}$ revealed a distinct and significant downregulation in CA1, CA3 and DG. We therefore speculate that this change in receptor distribution may be GluA2-mediated beginning as early as P14 in the CA3, with sequential temporal downregulation in other regions, as Schaeffer collaterals make connections with CA1, and DG neurogenesis is completed. Regional CA pyramidal or DG granule cell number, hippocampal volume, or cell density was not affected at this time point or in adults. 3,5

PNE downregulates NMDAR expression in adulthood, likely affecting the amplitude and frequency of NMDAmediated responses, ${ }^{42}$ but the current findings suggest that the impact of PNE on transmission through the NMDA receptor is already detectable by the infancy-equivalent age of P14. Furthermore, the finding of reduced GluN2B could indicate premature maturation of glutamatergic synapses combined with reduced inputs to hippocampal interneurons ${ }^{43}$ to modulate hippocampus-mediated behaviors such as learning and memory-associated tasks. Another pivotal neurodevelopmental period in which PNE exerts its dysregulation on NMDAR likely exists, as previous data shows a reduction in adult levels of NMDAR due to GluN1 downregulation, for which regional specificity is not yet detectable at P14.

We find here that CaMKII $\alpha$ is repressed specifically in the CA3 and DG, a finding which was likely masked when the whole P14 hippocampal formation was dissected and analyzed. ${ }^{29}$ In this study, we provide a better spatial resolution of regional variations in synaptic expression. Of the CaMKII subunits, CaMKII $\alpha$ displays highly specific cellular and subcellular distribution and pattern of expression exclusively at excitatory synaptic contacts in neuronal populations. ${ }^{44,45}$ The predominance of excitatory $v$. inhibitory presynaptic neuronal inputs would directly affect the expression of CaMKII $\alpha$. Thus, both nicotine perturbation of glutamatergic neurotransmission and the basal levels of excitatory $v$. inhibitory projections may play a role in CaMKII $\alpha$ expression such that subregional differences reflect the variability in inputs with greater fidelity than the whole homogenate of hippocampus. These data suggest that the CA3 and DG may be more sensitive to gestational nicotine exposure than other subregions of the hippocampal formation, including CA1. Furthermore, CaMKII $\alpha$ disruption may precede that of glutamate receptors as DG development is delayed compared with CA regions. CaMKII $\alpha$ is responsible for glutamate receptor phosphorylation and insertion into the membranes is necessary for LTP induction, is persistently activated by stimuli that elicit LTP, and can, by itself, enhance the efficacy of synaptic transmission. ${ }^{13}$ Therefore, decreased expression of CaMKII $\alpha$ may be responsible for the dysregulation or impairment of these functions. In addition, since the DG is well documented as a site of adult neurogenesis, the repression of CAMKII $\alpha$ early in neurodevelopment may therefore also adversely impair the efficiency of synaptic 
transmission possibly altering the strength of connections of newly forming neurons.

Previously, when whole hippocampal homogenate was analyzed, SNAP25 was elevated at P1, but had stabilized and showed no modulation from control at P14 or $\mathrm{P}^{2}{ }^{29}$ The present study reveals a significant increase in levels of SNAP25 maintained in the P14 CA3. This data underscores the significance of the microanatomical analyses in this investigation to isolate subregional differences that are not detectable in the whole homogenate. An increase in SNAP25 indicates possible exocytic activity due to nicotine mediated glutamate release which may in turn disrupt CA3 functioning, namely, the encoding of context-dependent memories, including extinction memories. ${ }^{46}$

Aside from modulating the components of the glutamatergic synapse itself, impaired nicotinic modulation of hippocampal circuitry has been proposed as a mechanism by which maternal nicotine exposure induces memory impairments and cognitive dysfunction. A recent study suggests that memory deficits in object recognition or retention tasks may be due to a reduced cholinergic modulation of Schaffer collaterals, for learning and memory, but not necessarily CA1 neuron LTP responses per se. ${ }^{8}$ Whereas the mechanisms of PNE-mediated learning deficits are likely more complex, this study strongly supports that CA3 neurons, from which Schaeffer collaterals originate, have impaired glutamatergic synaptic signaling.

In conclusion, the current study reinforces previous findings that PNE dysregulates glutamatergic receptor stoichiometry at pivotal neurodevelopmental time points and proposes that gestational nicotine exposure may result in hippocampal dysfunction as early as P14. Studies analyzing effects of gestational nicotine or other early stressors on adolescent and adult behaviors must consider regional differences, which can be adjacent, but functionally and anatomically distinct. This study reports disruption of synaptic processing associated disproportionately with the $\mathrm{CA} 3$, a major area of converging and diverging inputs in the hippocampus.

\section{Acknowledgment}

The authors thank Dr Hong Wang for his assistance.

\section{Financial Support}

This research was supported, in part, by funding from the National Institutes of Health, Grants \#AA021262, \#AA021262S1 and \#MD007597.

\section{Conflict of Interests}

None.

\section{Ethical Standards}

The authors assert that all procedures contributing to this work comply with the ethical standards of the Guide for the Care and
Use of Laboratory Animals. This study was approved by the Howard University Institutional Animal Care and Use Committee (IACUC).

\section{References}

1. Tobacco Use and Pregnancy. A Report by the Pregnancy Risk and Monitoring System. U.S. Department of Health and Human Services. Centers for Disease Control and Prevention (CDC). (PRAMS), 2011 . Retrieved 15 August 2015 from http://www.cdc. gov/reproductivehealth/maternalinfanthealth/tobaccousepregnancy/ index.htm.

2. Parameshwaran K, Buabeid MA, Bhattacharya $S$, et al. Long term alterations in synaptic physiology, expression of $\beta 2$ nicotinic receptors and ERK1/2 signaling in the hippocampus of rats with prenatal nicotine exposure. Neurobiol Learn Mem. 2013; 106, 102-111.

3. Wang H, Gondré-Lewis MC. Prenatal nicotine and maternal deprivation stress de-regulate the development of CA1, CA3, and dentate gyrus neurons in hippocampus of infant rats. PLoS One. 2013; 8, e65517.

4. Vaglenova J, Parameshwaran K, Suppiramaniam V, et al. Long-lasting teratogenic effects of nicotine on cognition: gender specificity and role of AMPA receptor function. Neurobiol Learn Mem. 2008; 90, 527-536.

5. Chen WJ, King KA, Lee RE, Sedtal CS, Smith AM. Effects of nicotine exposure during prenatal or perinatal period on cell numbers in adult rat hippocampus and cerebellum: a stereology study. Life Sci. 2006; 79, 2221-2227.

6. Abdel-Rahman A, Dechkovskaia AM, Sutton JM, et al. Maternal exposure of rats to nicotine via infusion during gestation produces neurobehavioral deficits and elevated expression of glial fibrillary acidic protein in the cerebellum and CA1 subfield in the offspring at puberty. Toxicology. 2005; 209, 245-261.

7. Roy TS, Seidler FJ, Slotkin TA. Prenatal nicotine exposure evokes alterations of cell structure in hippocampus and somatosensory cortex. J Pharmacol Exp Ther. 2002; 300, 124-133.

8. Nakauchi S, Malvaez M, Su H, et al. Early postnatal nicotine exposure causes hippocampus-dependent memory impairments in adolescent mice: association with altered nicotinic cholinergic modulation of LTP, but not impaired LTP. Neurobiol Learn Mem. 2015; 118, 178-188.

9. Xavier GF, Oliveira-Filho FJ, Santos AM. Dentate gyrus-selective colchicine lesion and disruption of performance in spatial tasks: Difficulties in 'place strategy' because of a lack of flexibility in the use of environmental cues? Hippocampus. 1999; 9, 668-681.

10. Moser EI. Altered inhibition of dentate granule cells during spatial learning in an exploration task. J Neurosi. 1996; 16, 1247-1259.

11. Gross CG. Neurogenesis in the adult brain: death of a dogma. Nat Rev Neurosci. 2000; 1, 67-73.

12. Altman J. Are new neurons formed in the brains of adult mammals. Science. 1962; 135, 1127-1128.

13. de Souza Silva MA, Huston JP, Wang AL, Petri D, Chao OY. Evidence for a specific integrative mechanism for episodic memory mediated by AMPA/kainate receptors in a circuit involving medial prefrontal cortex and hippocampal CA3 region. Cereb Cortex. 2015; 26, 3000-3009. 
14. Jensen O, Lisman JE. Hippocampal CA3 region predicts memory sequences: accounting for the phase precession of place cells. Learn Mem. 1996; 3, 279-287.

15. Levy WB. A sequence predicting CA3 is a flexible associator that learns and uses context to solve hippocampal-like tasks. Hippocampus. 1996; 6, 579-590.

16. Hunsaker MR, Lee B, Kesner RP. Evaluating the Temporal context of episodic memory: the role of CA3 and CA1. Behav Brain Res. 2008; 188, 310-315.

17. Farovik A, Dupont LM, Eichenbaum H. Distinct roles for dorsal CA3 and CA1 in memory for sequential nonspatial events. Learn Mem. 2009; 17, 12-17.

18. Hoge J, Kesner RP. Role of CA3 and CA1 subregions of the dorsal hippocampus on temporal processing of objects. Neurobiol Learn Mem. 2007; 88, 225-231.

19. Manns JR, Howard MW, Eichenbaum H. Gradual changes in hippocampal activity support remembering the order of events. Neuron. 2007; 56, 530-540.

20. Amaral DG, Ishizuka N, Claiborne B. Neurons, numbers and the hippocampal network. Prog Brain Res. 1990; 83, 1-11.

21. Witter MP. Intrinsic and extrinsic wiring of CA3: indications for connectional heterogeneity. Learn Mem. 2007; 14, 705-713.

22. Sik A, Ylinen A, Penttonen M, Buzsáki G. Inhibitory CA1-CA3hilar region feedback in the hippocampus. Science. 1994; 265, $1722-1724$.

23. Tsien JZ, Huerta PT, Tonegawa S. The essential role of hippocampal CA1 NMDA receptor-dependent synaptic plasticity in spatial memory. Cell. 1996; 87, 1327-1338.

24. Wong RW, Setou M, Teng J, Takei Y, Hirokawa N. Overexpression of motor protein KIF17 enhances spatial and working memory in transgenic mice. Proc Natl Acad Sci USA. 2002; 99, 14500-14505.

25. Malinow R, Malenka RC. AMPA receptor trafficking and synaptic plasticity. Annu Rev Neurosci. 2002; 25, 103-126.

26. Kessels HW, Malinow R. Synaptic AMPA receptor plasticity and behavior. Neuron. 2009; 61, 340-350.

27. Riedel G, Micheau J, Lam AG, et al. Reversible neural inactivation reveals hippocampal participation in several memory processes. Nat Neurosci. 1999; 2, 898-905.

28. Sanderson DJ, McHugh SB, Good MA, et al. Spatial working memory deficits in GluA1 AMPA receptor subunit knockout mice reflect impaired short-term habituation: evidence for Wagner's dual-process memory model. Neuropsychologia. 2010; 48, 2303-2315.

29. Wang H, Dávila-García MI, Yarl W, Gondré-Lewis MC. Gestational nicotine exposure regulates expression of AMPA and NMDA receptors and their signaling apparatus in developing and adult rat hippocampus. Neuroscience. 2011; 188, 168-181.

30. Wang H, Gondré-Lewis MC. Prenatal nicotine and maternal deprivation stress de-regulate the development of CA1, CA3, and dentate gyrus neurons in hippocampus of infant rats. PLoS One. 2013; 8, e65517.
31. Lucchesi W, Mizuno K, Giese KP. Novel insights into CaMKII function and regulation during memory formation. Brain Res Bull. 2011; 85, 2-8.

32. Altman J, Bayer SA. Migration and distribution of two populations of hippocampal granule cell precursors during the perinatal and postnatal periods. J Comp Neurol. 1990; 301, 365-381.

33. Harris KM, Jensen FE, Tsao B. Three-dimensional structure of dendritic spines and synapses in rat hippocampus (CA1) at postnatal day 15 and adult ages: implications for the maturation of synaptic physiology and long-term potentiation. $J$ Neurosci. 1992; 12, 2685-2705, Erratum in: J Neurosci 1992; 12(8).

34. Han G, An L, Yang B, Si L, Zhang T. Nicotine-induced impairments of spatial cognition and long-term potentiation in adolescent male rats. Hum Exp Toxicol. 2014; 33, 203-213.

35. Jia N, Yang K, Sun Q, et al. Prenatal stress causes dendritic atrophy of pyramidal neurons in hippocampal CA3 region by glutamate in offspring rats. Dev Neurobiol. 2010; 70, 114-125.

36. Scharfman HE. The CA3 'backprojection' to the dentate gyrus. Prog Brain Res. 2007; 163, 627-637.

37. Risold PY, Swanson LW. Connections of the rat lateral septal complex. Brain Res Rev. 1997; 24, 115-195.

38. Gaykema RP, van der Kuil J, Hersh LB, Luiten PG. Patterns of direct projections from the hippocampus to the medial septumdiagonal band complex: anterograde tracing with Phaseolus vulgaris leucoagglutinin combined with immunohistochemistry of choline acetyltransferase. Neuroscience. 1991; 43, 349-60.

39. Amaral DG, Witter MP. The hippocampal formation. In The Rat Nervous System (ed. Paxinos, G), 1995; pp 443-493. Academic Press: San Diego.

40. Gondré-Lewis MC, Warnock K, June HL Jr, et al. Binge drinking and impulsivity after maternal deprivation stress is mediated through TLR4 and alpha-1 and alpha-2 GABA receptors. Stress. 2016; 19, 235-247.

41. Gondré-Lewis MC, Darius PJ, Allard J. Stereological analyses of reward system nuclei in maternally deprived/separated alcohol drinking rats. J Chem Neuroanat. 2016; 76 (Pt B), 122-132.

42. Li J, Bo L, Zhang P, Gao Q, et al. Exposure to nicotine during pregnancy and altered learning and memory in the rat offspring. Nicotine Tob Res. 2015; 17, 661-666.

43. Kelsch W, Li Z, Wieland S, et al. GluN2B-containing NMDA receptors promote glutamate synapse development in hippocampal interneurons. J Neurosci. 2014; 34, 16022-16030.

44. Liu XB, Murray KD. Neuronal excitability and calcium/ calmodulin-dependent protein kinase type II: location, location, location. Epilepsia. 2012; 53, 45-52.

45. Liu XB, Jones EG. Alpha isoform of calcium calmodulin dependent protein kinase II (CAM II kinase- $\alpha$ ) restricted to excitatory synapses in the CA1 region of rat hippocampus. NeuroReport. 1997; 8, 1475-1479.

46. Kalisch R, Korenfeld E, Stephan KE, et al. Context-dependent human extinction memory is mediated by a ventromedial prefrontal and hippocampal network. J Neurosci. 2006; 26, 9503-9511. 